\title{
Robust Control Design for Positioning of an Unactuated Surface Vessel
}

\author{
Baris Bidikli, Enver Tatlicioglu and Erkan Zergeroglu
}

\begin{abstract}
In this paper, a robust controller is designed to achieve accurate positioning of an unactuated surface vessel by using multiple unidirectional tugboats. After initially locating opposing tugboats to specific configurations, the control problem is transformed into a second order system with an uncertain non-symmetric input gain matrix. Upon applying a matrix decomposition, a robust controller is proposed. Detailed stability analysis ensured asymptotic tracking. Numerical simulation results demonstrate the efficiency of the proposed controller.
\end{abstract}

\section{INTRODUCTION}

The positioning of large surface vessels like barges, platforms and unactuated ships is considered as the marine example of the swarm robotic applications. Since these types of large vessels can not generate necessary position and orientation control effort due to their low speed operation, they are in need of assistance of multiple tugboats. As a result of this, this objective is realized via a group of tugboats that are strategically positioned along the vessel's hull. Manipulation with multiple tugboats can be seen as a feasible solution for these type of applications. However, the radio communication between all involved tugboats, especially in human manipulated tugboats, affects the control performance dramatically. Although, the communication performance is increased with advanced global positioning systems, control of these type of systems is still a challenging problem because of possible problems that may arise in communication system during manipulation. As a natural result of these, above mentioned marine control problem has attracted attention of the control community.

In the last decade, different types of controller designs have been proposed for these type of applications. In [1], orientation tracking control of a unactuated vessel through the utilization of a swarm of vehicles operating in a decentralized fashion was achieved via presented robust control strategy. In order to design this robust controller, the influence of the other swarm vehicle was treated as a force disturbance into the model dynamics. In [2], an exact model knowledge position and orientation tracking controller was proposed for an unactuated surface vessel. In [3], an adaptive position

B. Bidikli is with the Department of Mechatronics Engineering, Izmir Katip Celebi University, 35620, Izmir, Turkey (Email: baris.bidikli@ikc.edu.tr).

E. Tatlicioglu is with the Department of Electrical \& Electronics Engineering, Izmir Institute of Technology, 35430, Izmir, Turkey (Phone: +90 (232) 7506536; Fax: +90 (232) 7506599; E-mail: enver@iyte.edu.tr).

E. Zergeroglu is with the Department of Computer Engineering, Gebze Technical University, 41400, Gebze, Kocaeli, Turkey (Email: ezerger@bilmuh.gyte.edu.tr).

E. Tatlicioglu and E. Zergeroglu are funded by The Scientific and Technological Research Council of Turkey via grant number 113E147.

All the correspondence should be addressed to E. Tatlicioglu. control strategy was designed without using the location of the tugboats about the vessel hull. In this paper, the control strategy was developed such that it does not require the communicating of data between tugboats. Another adaptive control strategy was presented in [4] that took the uncertainty of system parameters into account. In [5], an optimization based force/torque allocation was employed and compared against a commutation based force/torque allocation strategy. Recently, in [6] a position tracking control of ship berthing with assistance of autonomous tugboats was provided by using sliding mode control approach.

In this paper, a robust control design for a position tracking of a large surface vessel manipulated by 6 autonomous tugboats is presented. First, the dynamic model of a 3 degree of freedom unactuated surface vessel manipulated by 6 unidirectional tugboats is given. Upon specification of the initial configurations of the uni-directional tugboats, the control is considered to be performed by 3 bi-directional tugboats. Next, the open-loop error system is obtained where an uncertain input gain matrix, which includes uncertain inertia matrix of the surface vessel and another uncertain matrix that includes positions of tugboats, is obtained. A matrix decomposition is applied to initialize the control design. A robust controller, which does not require the configurations of the 3 bi-directional tugboats, is then proposed. Detailed stability analysis is presented where asymptotic tracking is ensured. Numerical simulations are performed where the positions and orientations of the tugboats are perturbed with sinusoidal terms and satisfactory tracking performance is obtained.

\section{SYSTEM MODEL}

The dynamic model of a 3 degree of freedom unactuated vessel manipulated by six external tugboats can be written as [7], [8], [9], [10]

$$
\begin{aligned}
M_{s} \dot{\nu}+D_{s} & =F \\
\dot{x} & =R \nu
\end{aligned}
$$

where $F=\left[F_{x}, F_{y}, M_{z}\right]^{\mathrm{T}} \in \mathbb{R}$ represents the total forces and moments applied to the vessel and acting on the vessels center of mass. The inertia matrix that includes such effects as added mass is denoted by $M_{s}(\nu) \in \mathbb{R}^{3 \times 3}$ and obtained as [7]

$$
M_{s}=M_{R B}+M_{A}
$$

where $M_{R B}(\nu) \in \mathbb{R}^{3 \times 3}$ denotes the positive definite, symmetric rigid body part of the inertia matrix while the added mass inertia matrix is represented by $M_{A}(\nu) \in \mathbb{R}^{3 \times 3}$. It should be noted that $M_{A}(\nu)$ is not necessarily symmetric 
that can result with an asymmetric $M_{s}(\nu)$ [7]. However, it is assumed that this added mass term does not lead a rank deficiency in $M_{s}(\nu)$ [i.e., $M_{s}(\nu)$ is full rank]. The matrix $D_{s}(\nu) \in \mathbb{R}^{3}$ contains drag, damping, and other parasitic effects while $x(t)=\left[x_{p}(t), y_{p}(t), \psi(t)\right]^{\mathrm{T}} \in \mathbb{R}^{3}$ represents the composite inertial position $\left(x_{p}, y_{p}\right)$ and heading $\psi$ of the vessel while body fixed linear and angular velocity signals are represented by $\nu(t)=[u(t), v(t), \dot{\psi}(t)]^{\mathrm{T}} \in \mathbb{R}^{3}$. The rotation matrix is denoted by $R(\psi) \in S O(3)$ and defined as

$$
R(\psi)=\left[\begin{array}{lll}
\cos (\psi) & -\sin (\psi) & 0 \\
\sin (\psi) & \cos (\psi) & 0 \\
0 & 0 & 1
\end{array}\right]
$$

\section{A. Force Decomposition and Commutation Strategy}

It is assumed that, the unactuated vessel is moved via thrust inputs that are provided from six tugboats in contact with the vessel's hull as shown in Figure 1. According to this assumption $F$ is a result of the combined efforts that is provided from six tugboats and expressed as

$$
F=B_{1} U_{1}
$$

where $U_{1}(t)=\left[u_{1 a}, u_{1 b}, u_{2 a}, u_{2 b}, u_{3 a}, u_{3 b}\right]^{\mathrm{T}} \in \mathbb{R}^{6}$ denotes a thrust input vector from six tugboats while the thrust configuration is shown by $B_{1} \in \mathbb{R}^{3 \times 6}$ is defined as

$$
B_{1}=\left[\begin{array}{lll}
1 & 0 & 0 \\
-1 & 0 & 0 \\
C_{\alpha 2 a} & S_{\alpha 2 a} & L_{y 2 a} C_{\alpha 2 a}-L_{x 2 a} S_{\alpha 2 a} \\
C_{\alpha 2 b} & S_{\alpha 2 b} & L_{y 2 b} C_{\alpha 2 b}-L_{x 2 b} S_{\alpha 2 b} \\
C_{\alpha 3 a} & S_{\alpha 3 a} & -L_{y 3 a} C_{\alpha 3 a}+L_{x 3 a} S_{\alpha 3 a} \\
C_{\alpha 3 b} & S_{\alpha 3 b} & -L_{y 3 b} C_{\alpha 3 b}+L_{x 3 b} S_{\alpha 3 b}
\end{array}\right]^{\mathrm{T}}
$$

where $C_{\theta}=\cos (\theta)$, and $S_{\theta}=\sin (\theta)$. The opposite tugboats can then be placed as follows for utilizing the unidirectional structure of tugboats

$$
\begin{array}{lll}
\alpha_{2 b}=\alpha_{2 a}+\pi & L_{x 2 b}=L_{x 2 a} & L_{y 2 b}=L_{y 2 a} \\
\alpha_{3 b}=\alpha_{3 a}-\pi & L_{x 3 b}=L_{x 3 a} & L_{y 3 b}=L_{y 3 a}
\end{array} .
$$

As a result of this, the force equation given in (5), the thrust

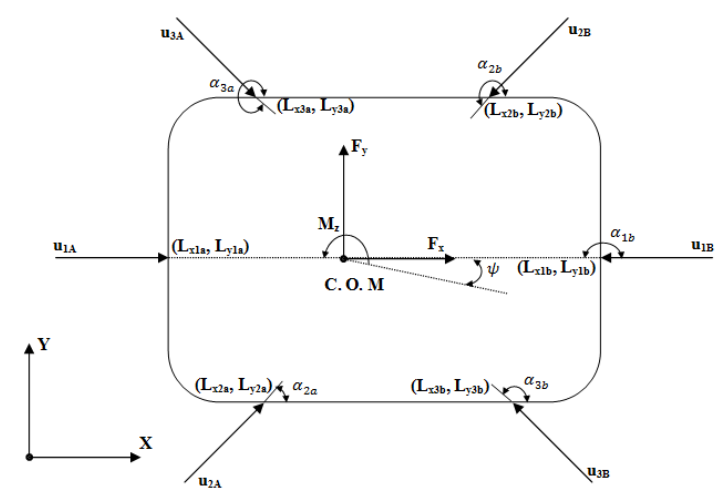

Fig. 1. System description showing the vessel frames

input vector and the thrust configuration matrix defined in same equation are obtained as

$$
\begin{aligned}
& F=B U \\
& U(t)=\left[u_{1}, u_{2}, u_{3}\right]^{\mathrm{T}} \\
& B=\left[\begin{array}{lll}
1 & 0 & 0 \\
C_{\alpha 2 a} & S_{\alpha 2 a} & L_{y 2 a} C_{\alpha 2 a}-L_{x 2 a} S_{\alpha 2 a} \\
C_{\alpha 3 a} & S_{\alpha 3 a} & -L_{y 3 a} C_{\alpha 3 a}+L_{x 3 a} S_{\alpha 3 a}
\end{array}\right]^{(10)}
\end{aligned}
$$

by utilizing the fact that $u_{i}=u_{i a}-u_{i b}, \forall i=1,2,3$.

Remark 1: Similar to [3] the tugboats are considered to be placed according to the configurations in (6). In this study, unlike [3] we consider the configurations in (6) are the initial configurations and that they may vary as the motion starts. This relaxes the static positioning assumption in [3] and is a novel departure from the existing literature.

The main purpose of the subsequent sections is to design $U(t)$ appropriately to obtain satisfactory tracking performance. The following commutation strategy can be applied to $U(t)$ to specify tugboat's unidirectional thrust effects [11]

$$
u_{i a}=\frac{1}{2}\left(u_{i}+\sqrt{u_{i}^{2}+\epsilon_{0}^{2}}\right) \quad u_{i b}=\frac{1}{2}\left(-u_{i}+\sqrt{u_{i}^{2}+\epsilon_{0}^{2}}\right)
$$

where $i=1,2,3$ and $\epsilon_{0} \in \mathbb{R}^{+}$denotes the control gain selected to obtain non-zero $u_{i j}(t)$ values that prevents the tugboats from losing contact with the vessel.

\section{B. Open-Loop Operation}

The time derivative of (2) is taken to obtain the following compact representation of the system

$$
\ddot{x}=\dot{R} \nu+R \dot{\nu}
$$

which contains the time derivative of $R(\psi)$ that can be obtained as follows because of the structure of the rotation matrix

$$
\dot{R}=R S_{3}
$$

where $S_{3}(\dot{\psi}) \in \mathbb{R}^{3 \times 3}$ is a skew-symmetric matrix defined as

$$
S_{3} \triangleq \dot{\psi}\left[\begin{array}{lll}
0 & -1 & 0 \\
1 & 0 & 0 \\
0 & 0 & 0
\end{array}\right]
$$

After substituting (13) into (12), the right hand side of (12) can be re-arranged as

$$
\ddot{x}=-R\left(M_{s}^{-1} D_{s}-S_{3} \nu\right)+R M_{s}^{-1} B U
$$

where (1) was utilized. The more compact form of the model is obtained as follows by rewriting the right-hand side of (15)

$$
\ddot{x}=h+G U
$$

where $h(x, \nu) \in \mathbb{R}^{3}$ and $G(x, \nu) \in \mathbb{R}^{3 \times 3}$ are defined as

$$
\begin{aligned}
h & \triangleq-R\left(M_{s}^{-1} D_{s}-S_{3} \nu\right) \\
G & \triangleq R M_{s}^{-1} B .
\end{aligned}
$$

Since $G$ is a real matrix with non-zero leading principal minors the following matrix decomposition is possible [12], [13]

$$
G=S D U_{u}
$$


where $S(x, \nu) \in \mathbb{R}^{3 \times 3}$ represents a symmetric positive definite matrix while a diagonal matrix with entries being \pm 1 and a unity upper triangular matrix are denoted by $D \in \mathbb{R}^{3 \times 3}$, and $U_{u}(x, \nu) \in \mathbb{R}^{3 \times 3}$, respectively. As a result of applying the above matrix decomposition to the models that are available in the literature, D came out to be an identity matrix. Despite this, the derivations given in this paper will be presented for the general case where it is assumed that $D$ is available for control design (see [14] for the presendence of this type assumption).

After taking the time derivative of (16), we obtain

$$
\dddot{x}=\varphi+S D U_{u} \dot{U}
$$

where (16) and (18) are utilized and an auxiliary signal, $\varphi(x, \dot{x}, \ddot{x}) \in \mathbb{R}^{3}$ is defined as

$$
\varphi \triangleq \dot{h}+\dot{G} G^{-1}(\ddot{x}-h) .
$$

At this point, we would like to define the inverse of $S$ as a new matrix denoted by $M(x, \nu) \in \mathbb{R}^{3 \times 3}$. It is remarked that $M$ is symmetric and positive definite because of symmetric and positive definite structure of $S$. In addition to this, the following bounds are valid for $M(x, \nu)$

$$
\underline{m}\|\chi\|^{2} \leq \chi^{T} M(x, \nu) \chi \leq \bar{m}(x, \nu)\|\chi\|^{2} \quad \forall \chi \in \mathbb{R}^{3}
$$

where $\underline{m} \in \mathbb{R}$ and $\bar{m}(x, \nu) \in \mathbb{R}$ are denoted a positive bounding constant and a positive non-decreasing function, respectively.

The following equation can be obtained by multiplying both sides of (19) with $M$

$$
M \dddot{x}=f+D U_{u} \dot{U}
$$

where $f(x, \dot{x}, \ddot{x}) \triangleq M \varphi \in \mathbb{R}^{3}$.

\section{ERror System DeVElopment}

Ensuring a good tracking performance for inertial positions and heading of the vessel for a given reference trajectory and the boundedness of all signals under the closed-loop operation constitute our main control objectives. The control design is based on availability of $x(t)$ and $\dot{x}(t)$ (i.e., full-state feedback).

The output tracking error, $e_{1}(t) \in \mathbb{R}^{3}$, is defined as follows to quantify the tracking control objective

$$
e_{1} \triangleq x_{d}-x
$$

where $x_{d}(t) \in \mathbb{R}^{3}$ is a smooth reference trajectory that is chosen in the sense that

$$
x_{d}(t) \in \mathcal{C}^{3} \text { and } x_{d}^{(i)}(t) \in \mathcal{L}_{\infty}, i=0,1,2,3 .
$$

In order to eliminate the higher order time derivatives from our Lyapunov-based stability analysis, the auxiliary error signals, $e_{2}(t) \in \mathbb{R}^{3}$ and $r(t) \in \mathbb{R}^{3}$ are defined as follows

$$
\begin{aligned}
e_{2} & \triangleq \dot{e}_{1}+e_{1} \\
r & =\dot{e}_{2}+\Gamma e_{2}
\end{aligned}
$$

where $\Gamma \in \mathbb{R}^{3 \times 3}$ denotes a positive-definite, diagonal, constant gain matrix. The following expression is obtained by taking time derivative of (26) and multiplying the result from left with $M$

$$
M \dot{r}=M\left(\dddot{x}_{d}+\ddot{e}_{1}+\Gamma \dot{e}_{2}\right)-f-D U_{u} \dot{U}
$$

where (22), and the time derivatives (23) and (25) were utilized. The right-hand side of (27) can be re-arranged as

$$
M \dot{r}=-\frac{1}{2} \dot{M} r-e_{2}-D U_{u} \dot{U}+N
$$

where $N\left(x, \dot{x}, \ddot{x}, x_{d}, \dot{x}_{d}, \ddot{x}_{d}, \dddot{x}_{d}, t\right) \in \mathbb{R}^{3}$ is an auxiliary function and defined as

$$
N \triangleq M\left(\dddot{x}_{d}+\ddot{e}_{1}+\Gamma \dot{e}_{2}\right)-f+e_{2}+\frac{1}{2} \dot{M} r
$$

The auxiliary function $N$ can be partitioned as a sum of two newly defined auxiliary signals denoted by $\bar{N}(t)$, $\widetilde{N}(t) \in \mathbb{R}^{3}$. These auxiliary signals are defined as

$$
\begin{aligned}
\bar{N} & \left.\triangleq N\right|_{x=x_{d}, \dot{x}=\dot{x}_{d}, \ddot{x}=\ddot{x}_{d}} \\
\widetilde{N} & \triangleq N-\bar{N} .
\end{aligned}
$$

After substituting the above definitions, the final form of open-loop error system can be obtained as follows by rearranging (28)

$$
M \dot{r}=-\frac{1}{2} \dot{M} r-e_{2}-D U_{u} \dot{U}+\widetilde{N}+\bar{N} .
$$

\section{Controller Formulation}

Motivated by the subsequent detailed stability analysis and based on the open-loop error system in (32), the control input $U(t)$ is designed as

$$
U=D K\left[e_{2}(t)-e_{2}\left(t_{0}\right)+\Gamma \int_{t_{0}}^{t} e_{2}(\sigma) d \sigma\right]+D \Pi
$$

where the auxiliary signal $\Pi(t) \in \mathbb{R}^{3}$ is generated according to the update law

$$
\dot{\Pi}(t)=C \operatorname{Sgn}\left(e_{2}(t)\right) \text { with } \Pi\left(t_{0}\right)=0_{3} .
$$

In (33) and (34), $K, C \in \mathbb{R}^{3 \times 3}$ denote positive definite, diagonal, constant gain matrices while a vector of zeros is represented by $0_{3} \in \mathbb{R}^{3}$ and $\operatorname{Sgn}(\cdot) \in \mathbb{R}^{3}$ is the vector signum function. The control gain is chosen as $K=I_{3}+k_{p} I_{3}+$ $\operatorname{diag}\left\{k_{d, 1}, k_{d, 2}, 0\right\}$ where $k_{p}, k_{d, 1}, k_{d, 2} \in \mathbb{R}$ are positive, constant controller gains, the notation $\operatorname{diag}\{\cdot\}$ represents a diagonal matrix, and $I_{3} \in \mathbb{R}^{3 \times 3}$ is the standard identity matrix. The following closed-loop error system is obtained by substituting the time derivative of (34) into (32) and then adding and subtracting $D K r(t)$

$$
\begin{aligned}
M \dot{r}= & -\frac{1}{2} \dot{M} r-e_{2}-K r+\tilde{N}+\bar{N} \\
& -D\left(U_{u}-I_{3}\right) D K r-D U_{u} D C \operatorname{Sgn}\left(e_{2}\right)
\end{aligned}
$$

where the fact that $D D=I_{3}$ was utilized.

Before presenting the accompanying stability analysis, more detailed examination of the last two terms of (35) are given. The $D\left(U_{u}-I_{3}\right) D K r$ term can be rewritten as

$$
D\left(U_{u}-I_{3}\right) D K r=\left[\begin{array}{c}
\Lambda_{1} \\
\Lambda_{2} \\
0
\end{array}\right]+\left[\begin{array}{c}
\Phi_{1} \\
\Phi_{2} \\
0
\end{array}\right]
$$


where the auxiliary signals $\Lambda_{1}(t), \Lambda_{2}(t), \Phi_{1}(t), \Phi_{2}(t) \in \mathbb{R}$ are defined as

$$
\begin{aligned}
& \Lambda_{1} \triangleq d_{1} d_{2} k_{2} \widetilde{U}_{u_{1,2}} r_{2}+d_{1} d_{3} k_{3} \widetilde{U}_{u_{1,3}} r_{3} \\
& \Lambda_{2} \triangleq d_{2} d_{3} k_{3} \widetilde{U}_{u_{2,3}} r_{3} \\
& \Phi_{1} \triangleq d_{1} d_{2} k_{2} \bar{U}_{u_{1,2}} r_{2}+d_{1} d_{3} k_{3} \bar{U}_{u_{1,3}} r_{3} \\
& \Phi_{2} \triangleq d_{2} d_{3} k_{3} \bar{U}_{u_{2,3}} r_{3}
\end{aligned}
$$

with the following definitions of $\bar{U}_{u_{1,2}}(t), \bar{U}_{u_{1,3}}(t)$, $\bar{U}_{u_{2,3}}(t), \widetilde{U}_{u_{1,2}}(t), \widetilde{U}_{u_{1,3}}(t), \widetilde{U}_{u_{2,3}}(t) \in \mathbb{R}$

$$
\begin{aligned}
& \left.\bar{U}_{u_{i, j}} \triangleq U_{u_{i, j}}\right|_{x=x_{d}, \dot{x}=\dot{x}_{d}} \\
& \widetilde{U}_{u_{i, j}} \triangleq U_{u_{i, j}}-\bar{U}_{u_{i, j}}
\end{aligned}
$$

where $U_{u_{i, j}}(x, \nu) \in \mathbb{R}$ represent the entries of $U_{u}(x, \nu)$. From (29), it can be seen that $\Lambda_{2}(t)$ depends on $k_{3}$, and from (37) it is clear that, $\Lambda_{1}(t)$ depends on $k_{3}$ and $k_{2}$. From (39) and (40) it can also seen that, $\Phi_{1}(t)$ depends on $k_{3}$ and $k_{2}$ while $\Phi_{2}(t)$ depends on $k_{3}$.

On the other hand, the following decomposition can be applied to $D U_{u} D C \operatorname{Sgn}\left(e_{2}\right)$ term

$$
D U_{u} D C \operatorname{Sgn}\left(e_{2}\right)=\left[\Psi^{T}, 0\right]^{T}+\Theta
$$

where two newly defined auxiliary signals denoted by $\Psi(t) \in \mathbb{R}^{2}$ and $\Theta(t) \in \mathbb{R}^{3}$ have the following forms

$$
\begin{aligned}
{\left[\Psi^{T}, 0\right]^{T} } & =D\left(U_{u}-\bar{U}_{u}\right) D C \operatorname{Sgn}\left(e_{2}\right) \\
\Theta & \triangleq D \bar{U}_{u} D C \operatorname{Sgn}\left(e_{2}\right)
\end{aligned}
$$

where $\left.\bar{U}_{u}\left(x_{d}, \dot{x}_{d}\right) \triangleq U_{u}\right|_{x=x_{d}, \dot{x}=\dot{x}_{d}} \in \mathbb{R}^{3 \times 3}$ is a function of reference trajectory and its time derivative, and $\Psi_{i}(t) \in \mathbb{R}$, $i=1,2$ and $\Theta_{i}(t) \in \mathbb{R}, i=1,2,3$, are defined as

$$
\begin{aligned}
\Psi_{i} & =d_{i} \sum_{j=i+1}^{3} d_{j} C_{j} \widetilde{U}_{u_{i, j}} \operatorname{sgn}\left(e_{2, j}\right) \\
\Theta_{i} & =d_{i} \sum_{j=i}^{3} d_{j} C_{j} \bar{U}_{u_{i, j}} \operatorname{sgn}\left(e_{2, j}\right) .
\end{aligned}
$$

Remark 2: The following upper bounds can be developed by utilizing the Mean Value Theorem [15]

$$
\begin{aligned}
\|\widetilde{N}(\cdot)\| & \leq \rho_{\widetilde{N}}(\|z\|)\|z\| \\
\left\|\widetilde{U}_{i, j}(\cdot)\right\| & \leq \rho_{i, j}(\|z\|)\|z\|
\end{aligned}
$$

where $\rho_{\widetilde{N}}(\cdot), \rho_{i, j}(\cdot) \in \mathbb{R}$ are non-negative, globally invertible, non-decreasing functions of their arguments, and $z(t) \in \mathbb{R}^{9}$ is defined as

$$
z \triangleq\left[\begin{array}{lll}
e_{1}^{T} & e_{2}^{T} & r^{T}
\end{array}\right]^{T} .
$$

It can be seen from (30) and (41) that $\bar{N}(t)$ and $\bar{U}_{u_{i, j}}(t)$ can be upper bounded as [16]

$$
\begin{aligned}
\left|\bar{N}_{i}(t)\right| & \leq \zeta_{\bar{N}_{i}} \\
\left|\bar{U}_{u_{i, j}}(t)\right| & \leq \zeta_{\bar{U}_{u_{i, j}}}
\end{aligned}
$$

where $\zeta_{\bar{N}_{i}}, \zeta_{\bar{U}_{u_{i}},} \in \mathbb{R}$ are positive bounding constants. Based on (37)-(40), (46), (47), following upper bounds can be obtained for $i=1,2$

$$
\begin{aligned}
\left|\Lambda_{i}\right| & \leq \rho_{\Lambda_{i}}(\|z\|)\|z\| \\
\left|\Phi_{i}\right| & \leq \zeta_{\Phi_{i}}\|z\| \\
\left|\Psi_{i}\right| & \leq \rho_{\Psi_{i}}(\|z\|)\|z\|
\end{aligned}
$$

and the following upper-bound can also be obtained for $i=$ $1,2,3$

$$
\left|\Theta_{i}\right| \leq \zeta_{\Theta_{i}}
$$

where (48)-(52) were utilized. From (56), it is clear that $\|\Theta\| \leq \zeta_{\Theta}$ is provided for some positive bounding constant $\zeta_{\Theta} \in \mathbb{R}$, and from (53)-(55), we obtain

$$
\left|\Lambda_{i}\right|+\left|\Phi_{i}\right|+\left|\Psi_{i}\right| \leq \rho_{i}(\|z\|)\|z\|
$$

where $\rho_{i}(\|z\|) \in \mathbb{R} i=1,2$, are non-negative, globally invertible, non-decreasing functions satisfying

$$
\rho_{\Lambda_{i}}+\rho_{\Psi_{i}}+\zeta_{\Phi_{i}} \leq \rho_{i} .
$$

After this point, we can continue with the Lyapunovbased boundedness and convergence analysis of our robust controller design.

\section{Stability Analysis}

Proving the boundedness of the error signals under the closed-loop operation by using Lyapunov-type stability analysis is the first purpose of this section. Then, we will present a lemma and obtain an upper bound for the integral of the absolute values of the entries of the time derivative of $e_{2}(t)$ via this boundedness result. This upper bound will be utilized in another lemma to prove the non-negativity of a Lyapunov-like function. Finally, asymptotic stability of the overall closed-loop system will be proven by using the results of this lemma.

Theorem 1: (Boundedness proof) The controller in (33) and (34) guarantee the boundedness of the error signals in (23), (25) and (26) provided that the controller gains $k_{d, 1}, k_{d, 2}$ and $k_{p}$ are chosen large enough compared to the initial conditions of the system and the following condition is satisfied for the mathematical model in (1) and (2),

$$
\lambda_{\min }(\Gamma) \geq \frac{1}{2}
$$

where $\lambda_{\min }(\Gamma)$ is the minimum eigenvalue of the gain matrix $\Gamma$.

Proof: The most general case of the proof is given in Appendix A of [17]. The proof for the Theorem 1 can be obtained by using 2 instead of $n$ given in the mentioned study.

Lemma 1: Provided that $e_{2}(t)$ and $\dot{e}_{2}(t)$ are bounded, the following expression for the upper bound of the integral of the absolute value of the $i$-th entry of $\dot{e}_{2}(t) i=1,2,3$ can be obtained

$$
\int_{t_{0}}^{t}\left|\dot{e}_{2, i}(\sigma)\right| d \sigma \leq \kappa_{1}+\kappa_{2} \int_{t_{0}}^{t}\left|e_{2, i}(\sigma)\right| d \sigma+\left|e_{2, i}\right|
$$


where $\kappa_{1}, \kappa_{2} \in \mathbb{R}$ are some positive bounding constants.

Proof: Readers can refer to [18] for the proof.

Remark 3: As a result of the fact that $\bar{U}_{u}(t)$ being unity upper triangular, $\Theta(t)$ in (45) can be rewritten as

$$
\Theta=\left(I_{3}+\Omega\right) C \operatorname{Sgn}\left(e_{2}\right)
$$

where $\Omega(t) \triangleq D\left(\bar{U}_{u}-I_{3}\right) D \in \mathbb{R}^{3 \times 3}$ is a strictly upper triangular matrix. Since it is a function of the reference trajectory and its time derivatives, its entries, denoted by $\Omega_{i, j}(t) \in \mathbb{R}$, are bounded in the sense that

$$
\left|\Omega_{i, j}\right| \leq \zeta_{\Omega_{i, j}}
$$

where $\zeta_{\Omega_{i, j}} \in \mathbb{R}$ are positive bounding constants.

Lemma 2: Consider the term

$$
L \triangleq r^{T}\left[\bar{N}-\left(I_{3}+\Omega\right) C \operatorname{Sgn}\left(e_{2}\right)\right] .
$$

Provided that the entries of the control gain matrix $C$ are chosen to satisfy

$$
\begin{aligned}
& C_{3} \geq \zeta_{\bar{N}_{3}}\left(1+\frac{\kappa_{2}}{\Gamma_{3}}\right) \\
& C_{2} \geq\left(\zeta_{\bar{N}_{2}}+\zeta_{\Omega_{2,3}} C_{3}\right)\left(1+\frac{\kappa_{2}}{\Gamma_{2}}\right) \\
& C_{1} \geq\left(\zeta_{\bar{N}_{1}}+\zeta_{\Omega_{1,2}} C_{2}+\zeta_{\Omega_{1,3}} C_{3}\right)\left(1+\frac{\kappa_{2}}{\Gamma_{1}}\right)
\end{aligned}
$$

where $\Gamma_{i}$ for $i=1,2,3$ denotes the $i^{t h}$ diagonal element of $\Gamma$, then it can be concluded that

$$
\int_{t_{0}}^{t} L(\sigma) d \sigma \leq \zeta_{L}
$$

where $\zeta_{L} \in \mathbb{R}$ is a positive bounding constant defined as

$\zeta_{L} \triangleq \kappa_{1} \sum_{i=1}^{2} \sum_{j=i+1}^{3} \zeta_{\Omega_{i, j}} C_{j}+\kappa_{1} \sum_{i=1}^{3} \zeta_{\bar{N}_{i}}+\sum_{i=1}^{3} C_{i}\left|e_{2, i}\left(t_{0}\right)\right|$.

Proof: Reader can refers to [18] for the proof.

Theorem 2: (Asymptotic convergence proof) Given the dynamic model in (1) and (2), the controller of (33) and (34) ensures the tracking error signal $e_{1}(t)$ converges to origin asymptotically in the sense that

$$
\left\|e_{1}(t)\right\| \rightarrow 0 \text { as } t \rightarrow+\infty
$$

provided that $\Gamma$ is chosen to satisfy (59), the entries of $C$ are chosen to satisfy (64)-(66), and $k_{p}, k_{d, 1}, k_{d, 2}$ are chosen large enough.

Proof: The most general case of the proof is given in Appendix D of [17]. The proof for the Theorem 2 can be obtained by using $2,3, \lambda_{1}, \lambda_{2}, \lambda_{3}$ and $\Gamma$ instead of $n, m$, $\lambda_{2}, \lambda_{3}, \lambda_{4}$ and $\alpha$ given in the mentioned study, respectively.

Remark 4: The entries of the control gain matrix $C$ are required to satisfy (64) which depends on the constant upper bounds of uncertain system functions, and the entries of the control gain matrix $K$ are required to be chosen large enough compared to the initial conditions of the system. While this seems like a weakness of the controller, we will address this issue by utilizing the self-tuning strategy that we recently designed in [19] and [20] for the family of the controllers in [16] as an add-on to adjust the entries of $C$ and $K$.

\section{Vi. Simulation Results}

The performance of the proposed robust controller was demonstrated via numerical simulations. The ship model in (1) was utilized with the following inertia matrix [8]

$$
M_{s}(x)=\left[\begin{array}{lll}
m+X_{\dot{u}} & 0 & 0 \\
0 & m-Y_{\dot{v}} & m x_{g}-Y_{\dot{r}} \\
0 & m x_{g}-N_{\dot{v}} & n_{d}=m x_{g}-Y_{\dot{r}}
\end{array}\right] .
$$

In the above inertia matrix, constant terms $Y_{\dot{r}}$ and $N_{\dot{v}}$ are selected as

$$
Y_{\dot{r}}=0.0, N_{\dot{v}}=-1.0
$$

to reflect the effects of added mass which result in a non symmetric $^{1}$ inertia matrix. The detailed information about the damping matrix $D_{s}(\nu)$ can also be found in [8]. The reference position of the vessel was given as

$x_{d}(t)=\left[\begin{array}{lll}10 \sin (0.1 t)(\mathrm{m}) & 10 \cos (0.1 t)(\mathrm{m}) & -0.1 t(\mathrm{rad})\end{array}\right]^{T}$

with the initial positions $x(0)=\left[0.3,2.5,-\frac{\pi}{4}\right]^{T}$ and the initial velocities $v(0)=0_{3}$. The control gains $K$ and $C$ were obtained via a self-tuning strategy [19], [20] as

$$
K=\operatorname{diag}\{168.2,112.3,107.2\}, C=\operatorname{diag}\{3.2,6.4,2.3\}
$$

and the other control gains $\Gamma$ and $\epsilon_{0}$ are selected as

$$
\Gamma=\operatorname{diag}\{1,5\}, \epsilon_{0}=\sqrt{5} .
$$

In order to obtain a proper time-dependent nature for the tugboats' positions without losing their contact with vessel's hull, six tugboats were positioned at the following locations with respect to the vessel's center of mass.

$$
\begin{aligned}
& L_{1 a}=(-0.5,0.1 \sin (t)) \\
& L_{1 b}=(0.5,0.1 \sin (t)) \\
& L_{2 a}=(-0.25+0.5 \sin (t),-0.145) \\
& L_{2 b}=(-0.25+0.5 \sin (t), 0.145) \\
& L_{3 a}=(0.1 \sin (t), 0.145) \\
& L_{3 b}=(0.1 \sin (t),-0.145) .
\end{aligned}
$$

The incident angle of each tugboat with respect to the hull was selected as follows

$$
\begin{aligned}
& \alpha_{1 a}=(\pi / 180) \sin (t), \\
& \alpha_{1 b}=\pi-(\pi / 180) \sin (t), \\
& \alpha_{2 a}=\pi / 2+(\pi / 180) \sin (t), \\
& \alpha_{2 b}=\alpha_{2 a}+(\pi / 180) \sin (t), \\
& \alpha_{3 a}=3 \pi / 2+(\pi / 180) \sin (t), \\
& \alpha_{3 b}=\alpha_{3 a}-\pi+(\pi / 180) \sin (t) .
\end{aligned}
$$

The actual position tracking errors and the control inputs are shown in Figures 2 and 3, respectively. From Figure 2 it can be clearly seen that unactuated surface vessel tracked the desired composite inertial positions and heading successfully which means that our control objective was successfully met.

\footnotetext{
${ }^{1}$ In the other control approaches about this subject available in the literature, especially in [3] which can be considered as the closest work to ours, the inertia matrix was selected as a symmetric matrix (i.e. same values were selected for the terms $Y_{\dot{r}}$ and $N_{\dot{v}}$ ). In our study, this matrix was selected in non symmetric form and we obtained satisfactory tracking performance. This can be considered as an other important advantage of our controller.
} 

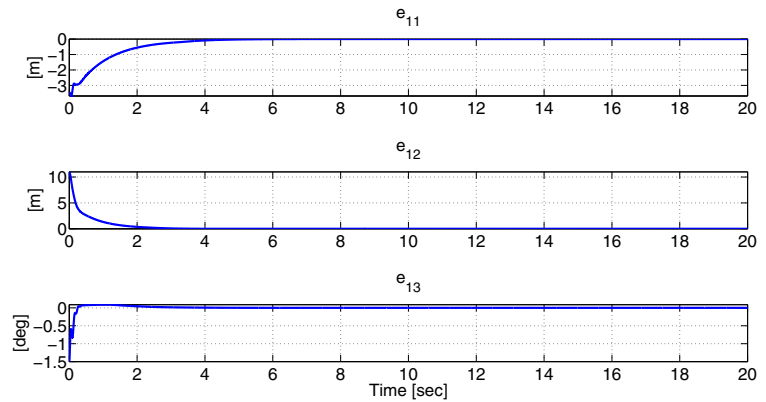

Fig. 2. Tracking Errors
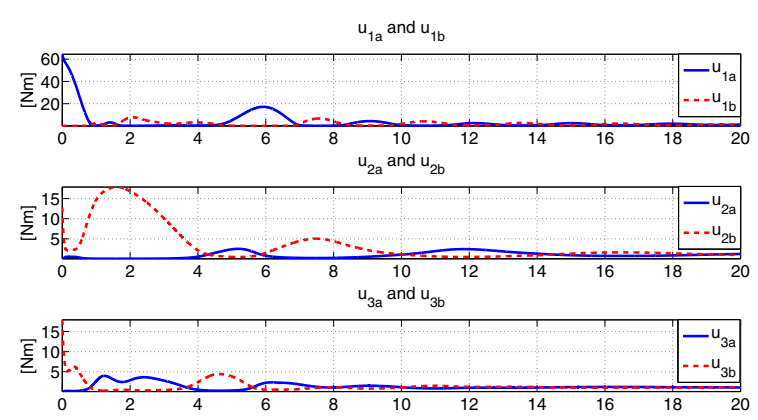

Fig. 3. Control Torques

\section{CONCLUSION}

In this paper, a robust controller for an unactuated surface vessel manipulated by autonomous tugboats was designed. The controller was designed under the restriction that the dynamic model of the surface vessel is uncertain. Furthermore, unlike the similar works in the literature, the surface vessel was considered to be under the influence of added mass effects which resulted in an asymmetric inertia matrix. The control problem is further complicated by the lack of accurate positions and orientations of tugboats. As a result of these issues, the resulting open-loop error system had an uncertain input gain matrix. A matrix decomposition method was applied and a robust controller was designed. The stability of the closed-loop system was investigated via detailed Lyapunov-type tools where asymptotic tracking was proven. Numerical simulations were performed where the positions and the orientations of the tugboats were considered to be disturbed by sinusoidal perturbations. Satisfactory tracking performance was obtained.

The novelties of the proposed control design and the accompanying stability analysis can be listed as:

- Different from the existing works on this application asymmetric inertial added mass effects were considered and dealt with.

- Positions and orientations of 6 unidirectional tugboats were considered to be available initially and then they were allowed to vary. This was demonstrated in the numerical simulations and can be seen from (75) and (76). It is noted that [3] did not allow positions and orientations of the tugboats to vary.

- A self-tuning strategy [19], [20] was employed as an add-on in the numerical simulations to ease the control gain tuning process.

Future work will focus on designing an optimal version of the proposed controller to optimize control efforts.

\section{REFERENCES}

[1] M. Feemster, J. Esposito, and J. Nicholson, "Manipulation of large objects by swarms of autonomous marine vehicles: Part i-rotation," in Proc. 38th Southeastern on Symposium on System Theory, Cooksville, TN, USA, 2006, pp. 255-259.

[2] E. Smith, M. Feemster, and J. Esposito, "Swarm manipulation of an unactuated surface vessel," in Proc. 39th Southeastern on Symposium on System Theory, Macon, GA, USA, 2007, pp. 16-20.

[3] D. Braganza, M. Feemster, and D. Dawson, "Positioning of large surface vessels using multiple tugboats," in Proc. American Control Conf., Times Square, NY, USA, 2007, pp. 912-917.

[4] V. Bui, H. Kawai, Y. Kim, and K. Lee, "A ship berting system design with four tug boats," J. of Mechanical Science and Technology, vol. 25, no. 5, pp. 1257-1264, 2010.

[5] J. Esposito, M. Feemster, and E. Smith, "Cooperative manipulation on the water using a swarm autonomous tugboats," in Proc. IEEE Int. Conf. Robot. Autom., Pasadena, CA, USA, 2008, pp. 1501-1506.

[6] V. Bui and Y. Kim, "Development of constrained control allocation for ship berthing by using autonomous tugboats," J. of Control, Automation and Systems, vol. 9, no. 6, pp. 1203-1208, 2011.

[7] T. I. Fossen, Guidance and Control of Ocean Vehicles. New York, NY, USA: John Wiley and Sons, 1994.

[8] _ - Marine Control Systems: Guidance, Navigation, and Control of Ships, Rigs and Underwater Vehicles. Trondheim, Norway: Marine Cybernetics AS, 2002.

[9] R. Skjetne, O. N. Smogeli, and T. I. Fossen, "A nonlinear ship manoeuvering model: Identification and adaptive control with experiments for a model ship," Modeling, Identification and Control, vol. 25, no. 1, pp. 3-27, 2004.

[10] F. Arrichiello, S. Chiaverini, and T. I. Fossen, "Formation control of underactuated surface vessels using the null-space-based behavioral control," in Proc. of International Conference on Intelligent Robots and Systems, 2006, pp. 5942-5947.

[11] M. S. de Queiroz and D. M. Dawson, "Nonlinear control of active magnetic bearings: A backstepping approach," IEEE Transactions on Control System Technology, vol. 4, no. 5, pp. 545-552, 1996.

[12] R. R. Costa, L. Hsu, A. K. Imai, and P. Kokotovic, "Lyapunov-based adaptive control of MIMO systems," Automatica, vol. 39, no. 7, pp. 1251-1257, 2003.

[13] A. S. Morse, "A gain matrix decomposition and some of its applications," Systems \& Control Letters, vol. 21, no. 1, pp. 1-10, 1993

[14] J. Chen, A. Behal, and D. M. Dawson, "Robust feedback control for a class of uncertain MIMO nonlinear systems," IEEE Trans. Autom. Control, vol. 53, no. 2, pp. 591-596, 2008.

[15] H. K. Khalil, Nonlinear Systems, 3rd Edition. New York, NY, USA Prentice Hall, 2002.

[16] B. Xian, D. M. Dawson, M. S. de Queiroz, and J. Chen, "A continuous asymptotic tracking control strategy for uncertain nonlinear systems," IEEE Trans. Autom. Control, vol. 49, no. 7, pp. 1206-1211, 2004.

[17] B. Bidikli, E. Tatlicioglu, E. Zergeroglu, and A. Bayrak, "An asymptotically stable continuous robust controller for a class of uncertain MIMO nonlinear systems," ArXiv e-prints, Tech. Rep. 1301.5483, Jan. 2013.

[18] B. Bidikli, E. Tatlicioglu, and E. Zergeroglu, "A robust tracking controller for dynamically positioned surface vessels with added mass," in Proc. IEEE Int. Conf. Decision and Control, Los Angeles, CA, USA, 2014, pp. 4385-4390.

[19] B. Bidikli, E. Tatlicioglu, A. Bayrak, and E. Zergeroglu, "A new robust 'integral of sign of error' feedback controller with adaptive compensation gain," in Proc. IEEE Int. Conf. Decision and Control, Florence, Italy, 2013, pp. 3782-3787.

[20] B. Bidikli, E. Tatlicioglu, and E. Zergeroglu, "A self tuning RISE controller formulation," in Proc. American Control Conf., Portland, OR, USA, 2014, pp. 5608-5613. 